\title{
Experimentação da técnica de narrativas OCC-RDD na prática. Um estudo de caso de uma aula no curso superior de ensino à computação.
}

\author{
Karina Buttignon ${ }^{1}$, Ítalo Santiago Vega ${ }^{1}$ \\ ${ }^{1}$ Pontifícia Universidade Católica - PUC-SP- Tecnologia da Inteligência e Design Digital \\ karina.buttignon@gmail.com, italo@pucsp.br
}

\begin{abstract}
This research aims to demonstrate in practice the OCC-RDD technique (Objective, mishap and Catastrophe, Reaction, Dilemma and Decision), discussed in the Study Group on Software Modeling (GEMS), under the Intelligence and Design Digital Technology Program PUC-SP. The paper presents the results of a case study of a class in the course of computing, using the concepts of OCC-RDD techniques, demonstrating new approaches to machine learning, using OCC-RDD narratives, can attack the student's cognitive difficulties. This is an intermediate step of a master's research, which has the specific objective of developing a authorship environment for development of OCC-RDD stories.
\end{abstract}

Resumo- Esta pesquisa tem como objetivo demonstrar na prática a técnica OCCRDD (Objetivo, Contratempo e Catástrofe, Reação, Dilema e Decisão), discutida no Grupo de Estudos em Modelagem de Software (GEMS), vinculado ao Programa de Tecnologia da Inteligência e Design Digital da PUC-SP. O trabalho apresenta os resultados de um estudo de caso de uma aula no curso de computação, usando os conceitos da técnicas OCC-RDD, demonstrando novas abordagens para aprendizagem computacional, usando narrativas OCC-RDD, podendo atacar as dificuldades cognitivas do estudante. Trata-se de uma etapa intermediaria de uma pesquisa de mestrado, que tem como objetivo específico desenvolver um ambiente de autorias para elaboração de histórias OCC-RDD.

\section{Introdução}

$\mathrm{Na}$ busca pelas melhores práticas pedagógicas, este trabalho descreve a técnica OCCRDD relacionada com a elaboração das cenas narrativas, como uma forma de atacar problemas cognitivos no curso de computação. A Técnica é um dos produtos do Grupo de Estudos em Modelagem de Software (GEMS) da PUC-SP, vinculado ao programa de pós-graduação Tecnologia da Inteligência e Design Digital (TIDD) e o objetivo deste artigo é apresentar um estudo de caso de uma aula de criptografia que ilustra um formato narrativo OCC-RDD.

A técnica OCC-RDD procura apresentar novas abordagens para a construção de ambientes de aprendizagem voltados a cursos de computação. Do ponto de vista da aprendizagem, acredita-se que uma técnica que pudesse "moldar" um encontro presencial dentro de um particular perfil cognitivo, minimizaria os efeitos de rendimento escolar e, consequentemente, motivaria o estudante a prosseguir nos seus estudos. Há relatos de diversas dificuldades no ensino-aprendizado na área de exatas, sobretudo se isso envolve abstração e raciocínio lógico. (PAZETO et al., 2014).

O uso da técnica OCC-RDD produz fábulas que ajudam na preparação de um ambiente de aprendizagem no qual podem ser incorporadas ferramentas e estratégias que possam 
incentivar o estudante a buscar conhecimento. Por exemplo, em cenas de "catástrofe" (não como um fator negativo de aprendizado, e sim, como uma forma dinâmica de rompimento de um determinado padrão de comportamento), o estudante é convidado a entrar em ação sobre a atividade desenvolvida. A participação dos personagens OCC-RDD que enfrentam o momento catastrófico é dirigida pelo mestre em comunhão com os estudantes, incentivando-os a realizar um pensamento individual e coletivo, simultaneamente.

As fábulas produzidas pelo uso da técnica são construídas por diversas cenas organizadas em dois grupos cognitivos: OCC - cenas narradas em terceira pessoa contemplando a apresentação de um objetivo a ser alcançado pelos personagens, seguido de cenas de contratempo e de catástrofe. Neste artigo, as cenas de contratempo e de catástrofe servem para o enquadramento dos elementos teóricos e práticos determinados pelo plano de ensino da disciplina. RDD - cenas narradas em primeira pessoa, procurando atuar diretamente com o estado emocional dos personagens, diante do problema a ser resolvido. Suas reações, dilemas e decisões na busca por resoluções dos problemas, são observados pelo mestre e pelos estudantes. Dentre as diferentes facetas que compõem o modelo de um personagem OCCRDD, destaca-se aquela que se fundamenta no modelo de personalidade, proposto por Mccrae \& John (1991) e que leva em consideração cinco fatores: estabilidade, comunicação, ponderação, conscienciosidade e intuição. Tais fatores também serão considerados no momento da construção de uma fábula de aula com personagens fictícios que apresentem personalidades assim modeladas, exemplificando suas emoções para aprender.

\subsection{A OCC-RDD e a Máquina de Estados}

A máquina de estados é uma forma de apresentar as ideias centrais da técnica OCC$\mathrm{RDD}$, por meio de roteiro semiautomático que possa orientar o mestre em suas aulas, apresentando uma fábula que irá auxiliá-lo durante a narração, podendo identificar diversas oportunidades de trilhas narrativas que podem ser exploradas e aplicadas. A Figura 1 mostra os dois ambientes da técnica OCC-RDD, no primeiro ambiente o mestre tem a opção através de uma máquina de estados de criar suas próprias histórias OCC-RDD e no segundo ambiente, mostra a história a ser narrada, usando a máquina de estados como apoio, neste caso, exemplificando um ambiente cujo propósito é desenvolver os conceitos de base a respeito da criptografia.

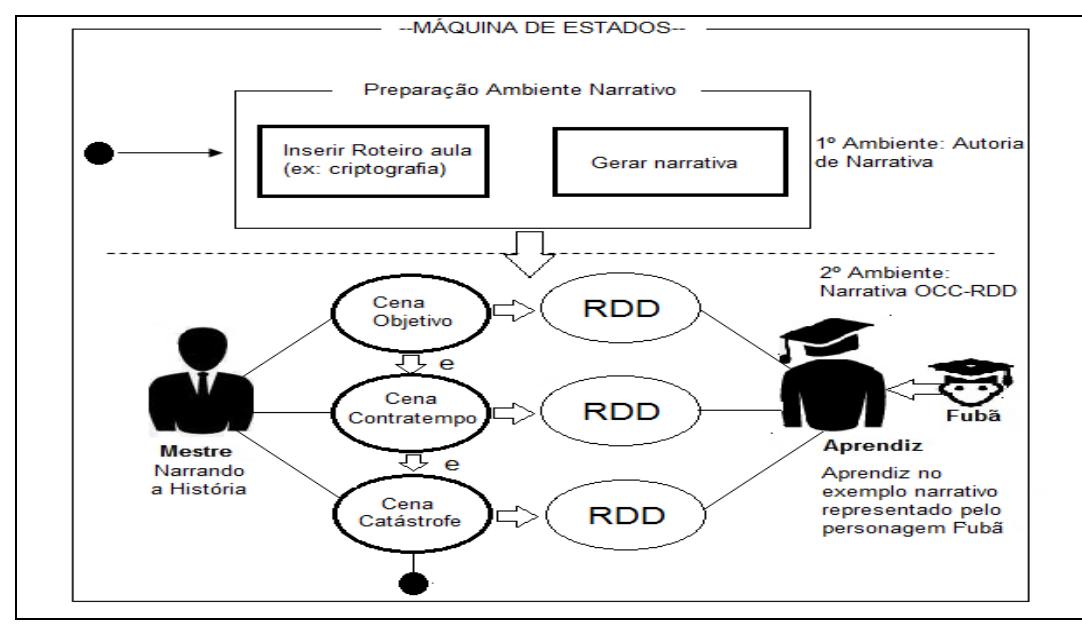

Figura 1- OCC-RDD e a Máquina de Estados

Fonte: Vega (2014)

A importância da técnica OCC-RDD para a elaboração de fábulas está ligada à maneira como o mestre irá introduzir o seu conteúdo de aula. A Figura 1 sugere a existência de uma 
interligação do estado da arte OCC-RDD, que no caso exemplifica com uma roteirização de aula de criptografia e em seguida a definição do texto da aula que será abordado. Têm-se então, na dramatização, dois participantes importantes para a composição desta aula: o mestre que irá narrar a história e o aprendiz, que são representados por personagens que compõe a história. No exemplo, representado pelo personagem "Fubã" que apresenta o seguinte arquétipo: estabilidade baixa; comunicação baixa; ponderação baixa; conscienciosidade alta; intuição baixa.

A máquina de estados, que é um modelo computacional, faz parte do mecanismo de geração automática de fábulas. Cada percurso nesta máquina conduz a trilhas de narração diferentes e dinamicamente estabelecidas dentro do ambiente de aprendizagem interativo. De certa forma, as trilhas em potencial guiam o mestre para as próximas cenas OCC-RDD. Na Figura 1, apresentam cenas "Objetivo", "Contratempo" e "Catástrofe", que geram no aprendiz "Reação", "Dilema" e "Decisão" para solucionar atividades em cada cena. O resultado é representado pela condição cognitiva do personagem "Fubã". A passagem de uma "cena", por exemplo, "Objetivo", para uma "cena", "Contratempo" é gerada a partir de um evento, representada pela letra "e". O evento é determinante para a tomada de decisão e será definido por um algoritmo para a mudança de cena, porém o evento está interligado ao ambiente, estendendo aos sentidos do professor em sala de aula.

\subsection{Identificação de Cenas Objetivo, Contratempo e Catástrofe}

Para melhor entendimento da técnica OCC-RDD é preciso detectar, dentro de um cenário narrativo de aula, quando a história passa de uma fase para outra, ou seja, encontrar características que possam definir quando dentro da narrativa está sendo referenciado a uma cena "objetivo", "contratempo", ou a uma "catástrofe". Como uma forma de identificar na narrativa essas cenas, a técnica OCC-RDD busca por meio das teorias da Taxonomia de Bloom, que define seis níveis de habilidades cognitivas, com características e conceitos, que possam identificá-las.

"Use of the taxonomy can also help one gain a perspective
on the emphasis given to certain behaviors by a particular
set of educational plans. Thus, a teacher, in classifying the
goals of a teaching unit, my find that they all fall within the
taxonomy category of recalling or remember in
knowledge". (BLOOM, 1984, p. 2).

$\mathrm{Na}$ Taxonomia de Bloom, os três níveis inferiores se apoia no reconhecimento e lembrança da informação, organização e ordenamento de material e a aplicação da informação aprendida. Já os três níveis superiores exigem que os alunos pensem crítica e criativamente, avaliem e transfiram habilidades e conhecimentos de uma situação para outra. (BATES \& MUNDAY, 2007, p.70). Considerando a teoria apresentada na Taxonomia de Bloom, esta pesquisa apresentará três momentos de uma aula de criptografia (estudo de caso), que identificam as cenas "Objetivo", "Contratempo" e "Catástrofe", segundo as características da Taxonomia:

Cenas Objetivo: tem como característica, determinar contextos e embasamentos teóricos diante de um tema a ser narrado em uma aula. Como por exemplo:

Disciplina: Aula de Redes de Computadores

Assunto: Criptografia

Aula 1: "Criptografia, considerada como a ciência e a arte de escrever mensagens em forma cifrada ou em código, é um dos principais mecanismos de segurança que você pode usar para se proteger dos riscos associados ao uso da Internet. Do grego: Cripto (escondido) + Grafia (ecrita)"; 
As definições sobre "criptografia" e a lembrança do significado da palavra "criptografia", define no exemplo de cena "objetivo", o "nível 1" na Taxonomia de Bloom, que elucida as competências e habilidades que o aprendiz precisa ter para a assimilar o que esta sendo proposto em aula. A habilidade de "Memorizar" ou "Lembrar" é uma característica comum dentro das cenas objetivo. Para Bates \& Munday (2007), o nível 1 da Taxonomia de Bloom é definido conforme a figura 2:

\begin{tabular}{|l|l|l|l|}
\hline Competência & Habilidades & Verbos úteis & Perguntas \\
\hline Conhecimento & Memorizar & Liste & Quem....? \\
& Lembrar & Identifique & O quê...? \\
& Reconnecer & Diga & Quando...? \\
& Relembrar & Defina & Onde...? \\
& & Classifique & Descreva o que \\
& & Nomeie & aconteceu... \\
& & Colecione & Quantos...? \\
\hline
\end{tabular}

Figura 2- Nível 1 da Taxonomia de Bloom Fonte: Bates \& Munday (2007)

Cenas de Contratempo: As cenas de contratempo tem como definição demonstrar por meio de narrativas, elementos textuais, cuja resposta é conhecida ou o caminho para a resposta é conhecido. O contratempo se baseia em atividades, como exercícios ou debates que façam o aprendiz a buscar informações que previamente foram aprendidas. Essa cena, é definida na Taxonomia de Bloom, também como nível 1, porém com caracterizadas por meio de exercícios que exija do aprendiz a busca pela contextualização já definida, a habilidade de "Lembrar", "Reconhecer" ou "Relembrar" são contundentes neste cenário. O exemplo 2 de uma aula de segurança com características de cenas de Contratempo:

\section{Disciplina: Aula de Redes de Computadores}

Assunto: Criptografia

Aula 2: Baseado nas informações sobre criptografia de descolamento por coluna usando chave, decifre o texto: SADEE MOOOS ABMCO ORSBJ EFERU VMOST - Chave: ZEBRAS

O exemplo 2 demonstra uma atividade com certo nível de dificuldade, pois o aprendiz precisa decifrar um texto criptografado, porém ele tem a lembrança, ou seja, um embasamento teórico definido previamente, onde explica o que é "criptográfica de descolamento por coluna usando chave", neste caso o aprendiz precisou recordar o que foi aprendido.

Cenas de Catástrofe: É definido como um estágio de amadurecimento. As atividades definidas nesta cena se classificam em um nível mais alto de abstração e geralmente o aprendiz não lembra ou não recorda a resposta. Os níveis de exercícios definidos para esta cena define que o aprendiz precisa ter compreensão para entender a situação e assim, tomar decisões que possam levar a um resultado satisfatório. Este cenário é definido na Taxonomia de Bloom como nível 2 e traz habilidades como: "Interpretar", "Organizar" e "Identificar". A figura 3 define o nível 2 da Taxonomia de Bloom.

\begin{tabular}{|c|c|c|c|}
\hline Competência & Habilidades & Verbos úteis & Perguntas \\
\hline Compreensão & $\begin{array}{l}\text { Interpretar } \\
\text { Organizar e } \\
\text { selecionar } \\
\text { Traduzir } \\
\text { de uma } \\
\text { linguagem } \\
\text { para outra } \\
\text { Identificar }\end{array}$ & $\begin{array}{l}\text { Resuma } \\
\text { Explique } \\
\text { Parafraseie } \\
\text { llustre } \\
\text { Avalie } \\
\text { Distinga } \\
\text { Preveja }\end{array}$ & $\begin{array}{l}\text { Você pode } \\
\text { recontar a } \\
\text { história...? } \\
\text { Você pode dar } \\
\text { um exemplo } \\
\text { de...? } \\
\text { Você pode } \\
\text { prever...? } \\
\text { Calcule } \\
\text { quantos...? }\end{array}$ \\
\hline
\end{tabular}

Figura 3- Nível 2 da Taxonomia de Bloom Fonte: Bates \& Munday (2007) 
O exemplo 3 de uma aula de segurança, com características de cenas de Catástrofe:

Disciplina: Aula de Redes de Computadores; Assunto: Criptografia

Aula 3: Crie um servidor de FTP com usuário anonymous e sem senha. Crie um texto criptografado e com esteganografia e copie para o diretório FTP do seu servidor. Crie um segundo diretório no seu servidor e coloque os arquivos de "prêmios" para aqueles que conseguirem acessar o servidor. Gere um usuário e senha - obs: o usuário e senha tem que estar no arquivo criptografado.

O exemplo 3 define uma atividade de criptografia, porém com um nível de abstração maior, elementos definidos no exercício como "Crie um servidor de FTP com usuário e senha", não estava previamente explicado na aula de criptografia, exigindo do aprendiz a busca por informações que não faziam parte do contexto.

Enfim, as cenas de Objetivo, Contratempo e Catástrofe são caracterizadas dentro da narrativa por elementos cognitivos fortemente discutidos na Taxonomia de Bloom. A técnica OCC-RDD se fundamenta nos dois níveis da taxonomia: o "conhecimento" e a "compreensão". Entende-se que a passagem para o terceiro nível da taxonomia de Bloom a "aplicação", seria um processo natural do aprendiz, considerando o seu nível de abstração através de todo embasamento teórico e prático, aprendido e exercitado no nível 1 e 2, a partir deste momento o aprendiz tem autonomia para usar as informações, métodos e conteúdos aprendidos em novas situações concretas.

\section{Discussão}

Com o objetivo de apresentar um novo modelo de aprendizado para as aulas no curso de computação, a técnica OCC-RDD tem seus princípios ligados à criação de uma fábula que possa estimular o aprendizado computacional principalmente nas disciplinas com maior dificuldade de aprendizado, como programação de computadores e disciplinas equivalentes.

Perraudeau (2006), em seu livro Estratégias de Aprendizagem comenta que a maioria de psicólogos, como Oliver Houdé (1998) distingue duas categorias de aprendizagem, algumas elementares enquanto que outras complexas. Ainda segundo Perraudeau (2006), para Houdé (1998), as primeiras dependem dos estímulos do meio, tais como diversas formas de condicionamentos; as segundas estão ligadas ao sentido, que o sujeito atribui aos estímulos.

A estrutura de fábulas OCC-RDD é tal que procura equilibrar os desafios, de forma que o humor, a motivação e o entusiasmo do estudante em aprender sejam positivos. Por exemplo, se o desafio é muito grande para a habilidade do estudante, a tendência é que a sua atitude em aprender diminua em função de stress excessivo. Por outro lado, quando o estudante apresenta uma habilidade muito grande em relação ao desafio apresentado, o efeito tende a ser o oposto: relaxamento e até mesmo o descaso pela atividade. A evolução do texto narrativo baseado na fábula OCC-RDD deve contemplar os níveis de conhecimento, para que possam propor situações que favoreçam estados de desafios e habilidade, evitando um stress excessivo e/ou relaxamento, buscando um caminho equilibrado de emoções, para que os estudantes com diferentes personalidades participem ativamente do ambiente de aprendizagem.

\section{1- Estudo de caso}

Para um entendimento prático da técnica OCC-RDD, com a finalidade de construir um roteiro de aula eficiente que desperte no aluno o raciocínio lógico de maneira natural e conciso e ao mesmo tempo em que gere prazer em aprender, este estudo de caso tem como objetivo demonstrar um meta-roteiro que servirá como uma proposta de narrativa e base para a construção de um roteiro de aula semiautomático usando da técnica OCC-RDD.

\subsubsection{Metodologia}

Para a elaboração do estudo de caso, foi utilizado da técnica qualitativa, com observação in loco dos estudantes pelo próprio pesquisador e coleta de dados através das 
opiniões dos participantes da amostra. A pesquisa ainda se encontra em pleno desenvolvimento e, portanto apenas resultados preliminares podem ser apresentados.

\subsubsection{1 - Método}

Pesquisa exploratória, realizada no curso de Analise e Desenvolvimento de Sistemas da Fatec Guaratinguetá-SP.

\subsubsection{2 - Amostra}

Participaram deste estudo 18 alunos estudantes de ambos o sexo, com idade entre 18 a 25 anos do curso de Análise e Desenvolvimento de Sistemas no $1^{\circ}$ semestre de 2015, da disciplina Redes de Computadores.

\subsubsection{3 - Instrumento}

Foram utilizadas aulas da disciplina de redes de computadores, abordando o mesmo tema do primeiro estudo de caso, a "criptografia", porém aplicado a uma nova turma, mas com a construção de uma narrativa que pudesse caracterizar a técnica OCC-RDD, utilizando de uma fábula, com personagens e lugares que caracterize a técnica. Essa atividade intercalou com fases de cenas "Objetivo", cenas de "Contratempo" e cenas de "Catástrofe".

\subsubsection{4- Procedimento}

Os sujeitos foram testados em um laboratório de informática. Solicitou-se que os alunos prestassem a atenção nas premissas iniciais da atividade proposta. A atividade se baseia em uma narrativa para solucionar uma frase criptografada, que exigirá dos alunos raciocínio lógico, conhecimento e compreensão para uma tomada de decisão eficiente. Para a aplicação da técnica OCC-RDD o mestre ao narrar à história, precisa criar "tramas", de assuntos abordados em aula, onde cada assunto se interliga ou necessita de informações anteriores. Para melhor entendimento da aplicação desse estudo de caso, o mesmo foi divido em "fases" a primeira e segunda fase, levou em média 70 minutos para o transcurso da aula, a terceira fase, em média 50 minutos, considerando a explicação e a realização da atividade conforme descritos a seguir:

$\mathrm{Na}$ primeira fase o mestre condutor do processo apresenta aos alunos o tema da aula "Criptografia", inserido cenas "Objetivo"; Porém é contada uma história, onde se apresenta personagens, Fubã, Espec, e Fê, em acontecimentos paralelos a aula, em situações em que o aluno em sala de aula se identifique com algum personagem e com o que é narrado. No primeiro momento a história é contada conforme o exemplo:

Durante a aula de Redes de Computadores, o aluno Fubã acessa a Internet e verifica seus emails, observa que recebeu um email desconhecido com uma mensagem estranha. Ao abri-la se depara como seguinte texto: HBMFSB $F$ P TFHVJOUF, UFOIP VN DPOWJUF B GBAFS: SSCGAPA! CCATUCOC AAEOAEH! RASGANIT RCPANENA HODRAARO UNOARRMN coxinha ;-)

Fubã não compreende nada do que esta contido no texto faz alguns testes mentais, mas é em vão, a mensagem é incompreensível. Volta a sua atenção a aula no momento em que o mestre discorre sobre o tema Criptografia com enfoque em Cifra de César.

Fubã fica interessado na matéria, mas a sua cabeça ainda esta tentando associar o que são aquelas letras que ele recebeu na mensagem....

Mostra pra Fê a mensagem, e qual foi a sua surpresa, ao perceber que Fê também recebeu a mensagem.

Mesmo intrigado Fubã retorna a atenção para a aula... Neste momento o mestre explica sobre criptografia:

Nesta primeira fase, a narrativa é contada através de um personagem que recebeu uma mensagem criptografada, porém Fubã não reconhece a regra de criptografia. O cenário inicial é uma cena de "Catástrofe", pois o personagem ao se deparar com uma mensagem criptografa, descobre que não tem condição de decodifica-la. A evidência inicial de uma cena de "Catástrofe", chama a atenção dos indivíduos que estão escutando a história que esta sendo narrada, ao mesmo tempo em que esta situação de decodificar a mensagem está intimamente 
interligada a outros "tramas" da aula. Sugere-se a elaboração de textos narrativos OCC-RDD, com conexões de assuntos, ou seja, para resolver a problema "C", precisa lembrar o assunto "A" ou "B".

Na segunda fase o mestre apresenta cenas "Objetivo" sobre teorias de criptografia, como exemplo:

Criptografia, considerada como a ciência e a arte de escrever mensagens em forma cifrada ou em código, é um dos principais mecanismos de segurança que você pode usar para se proteger dos riscos associados ao uso da Internet. Do grego: Cripto (escondido) + Grafia (escrita);E cita alguns exemplos como: Cifra de César.

A história continua sua narração e o personagem Fubã começa a associar a teoria apresentada "Cifra de César" com a mensagem criptografada recebida, e através de conceitos teóricos apresentados, a primeira parte da atividade de contratempo é decifrada.

Nesta fase da atividade o professor demonstra novamente a mensagem criptografada e debate com os alunos uma forma de decifrar. Os alunos associam a situação vivida por Fubã, com a sua condição em sala de aula e tentam decifrar a mensagem. O mestre neste momento tem opção de avançar a atividade para um evento de nível maior de aprendizagem, ou retornar as teorias para um melhor esclarecimento sobre o tema abordado. E a narrativa continua:

Fubã fica impressionado com o descolamento de letras, e rapidamente começa a pensar na mensagem recebida.

Fubã olha discretamente para a mensagem e consegue decifrar uma parte da mensagem:

HBMFSB F P TFHVJOUF, UFOIP VN DPOWJUF B GBAFS:

Galera é o seguinte, tenho um convite a fazer:

SSCGAPA! CCATUCOC AAEOAEH! RASGANIT RCPANENA HODRAARO UNOARRMN coxinha

Mas a outra parte da mensagem o deslocamento de letras não bate com a teoria do professor, rapidamente ele pergunta:

-Existem outras formas de criptografar?

O mestre apresenta a seus alunos em sala de aula uma situação onde por meio de conceitos teóricos a primeira parte da mensagem foi decifrada com sucesso, mas ainda, existe uma dificuldade em relação a segunda parte. Há uma atenção maior dos alunos em relação à aula, pois os alunos querem saber o que tem na segunda parte da mensagem. A aula transcorre com outras teorias de criptografia, exemplificando as cifras de "Bastão de Licurgo", "Descolamento por Transposição" e "Descolamento por transposição usando Chaves".

A sequência da narrativa mostra uma associação que "Fubã" e "Fê" fazem com o texto criptografado e o tema apresentado pelo professor "Descolamento por transposição usando Chaves". Usando a teoria apresentada pelo mestre, Fubã usa a técnica de descolamento usando chaves, consegue decifrar o restante da palavra, conforme a figura 4 :

\begin{tabular}{|l|l|l|l|l|l|l|}
\hline C & O & X & I & N & H & A \\
\hline C & H & U & R & R & A & S \\
\hline C & O & N & A & C & A & S \\
A & D & O & S & P & E & C \\
\hline T & R & A & G & A & O & G \\
U & A & R & A & N & A & A \\
\hline C & A & R & N & E & E & P \\
O & R & M & I & N & H & A \\
\hline C & O & N & T & A & ! & ! \\
\hline
\end{tabular}

Figura 4 - Atividade de criptografia - descolamento por transposição usando chaves Fonte: próprio autor 
A figura 4 mostra a montagem feita pelo personagem Fubã associando ao exemplo dado pelo mestre em aula. Em paralelo a narrativa, os alunos associam a situação vivida pela personagem e tenta decifrar a mensagem sugerida pelo professor em sala de aula. Este cenário remete a uma atividade de "Contratempo", pois alunos precisaram lembrar-se de elementos textuais previamente apresentado na aula de "criptografia". Os alunos conseguem decifrar aplicando a técnica de descolamento usando chaves, onde a tradução final desta segunda parte da mensagem pode ser lida horizontalmente, através da Figura 4: "Churrasco na casa do Spec traga o guaraná a carne e por minha conta!!"

$\mathrm{Na}$ terceira fase o professor segue com a narrativa desafiando os alunos com uma atividade com caraterísticas de "Catástrofe"; Aparecerá na narrativa elementos textuais que não foram tratados nesta aula, e os alunos terão que interpretar as informações para a resolução do problema.

Acredito que todos já foram convidados para o churrasco na casa do Spec, agora vamos ver se vocês são capazes de decifrar este exercício!

Fubã percebe que a mensagem que foi enviada por email no inicio da aula, partiu do professor e fica espantado: -Foi o professor que enviou a mensagem!!!

O mestre segue a sequencia da aula e pede que seus alunos agora execute a seguinte atividade:

- Acesse um servidor FTP e baixe um arquivo com criptografia e com esteganofria. Decifre o arquivo e procure mensagens que leve ao nome do usuário e senha de acesso ao servidor. (A senha tem 8 caracteres). Acesse o servidor e tenha acesso aos arquivos da aula.

Fubã e Fê se espantam pois eles não sabem como criar um servidor de FTP.

$O$ mestre recorda da aula de criação de servidor e a partir deste momento é possível dar continuidade ao exercício.

O nível da atividade foi caracterizado com um grau maior de dificuldade, pois exigia dos alunos conhecimentos em criptografia, montagem de servidor FTP, comandos de acesso e administração de arquivos. Foi considerada uma atividade de "Catástrofe", pois os alunos precisavam interpretar e recordar de informações para concluir a tarefa. O professor sugere que os grupos se dividam em dois lados "A" e "B" e criem seu próprio servidor de FTP, texto criptografado e formas de acessar, desafiando o outro grupo. Neste momento é apresentado um novo cenário, onde o aluno precisa exercitar todos os conhecimentos teóricos e práticos exercitados para uma tomada de decisão, despertando nos aprendizes "Reações, Dilemas e Decisões" para lidar com os novos desafios.

\subsubsection{5 - Resultado do Estudo de Caso}

Após a execução da atividade foi apresentado aos participantes da amostra um questionário que tinha como objetivo investigar se os alunos compreenderam a atividade e descobrir seu nível de dificuldade em relação à narrativa proposta é importante destacar que a atividade foi considerada difícil pelo professor. Na primeira parte da pesquisa foi perguntado aos participantes da amostra o nível de dificuldade da atividade, conforme o Gráfico 1.

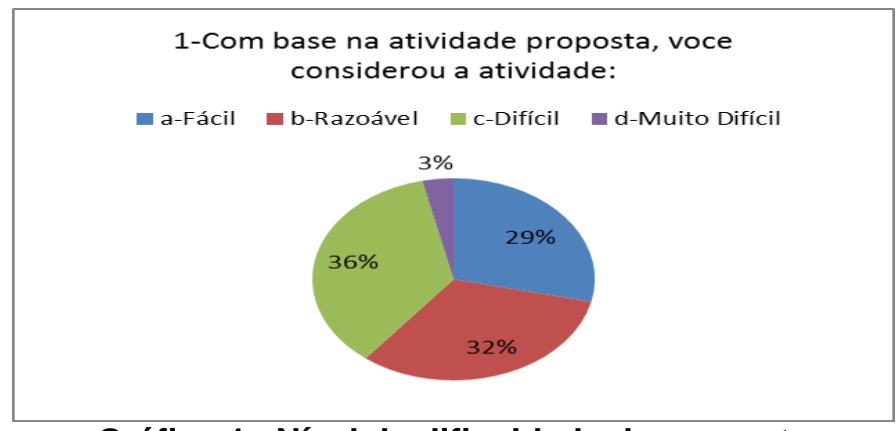

Gráfico 1- Nível de dificuldade da proposta

Fonte: próprio autor 
Conforme citado no Gráfico 1, 36\% dos participantes da atividade consideraram a atividade difícil, em concordância com a opinião do professor. Destacam-se também que 32\% dos alunos pesquisados acharam a atividade razoável, 29\% fácil e 3\% consideraram a atividade muito difícil.

Já a segunda parte da pesquisa fazia referência ao tempo médio que os participantes levaram para realizar a atividade final após a aula ser narrada. Segundo os apontamentos do Gráfico 2 a maioria levou mais de 30 minutos para resolver a atividade.

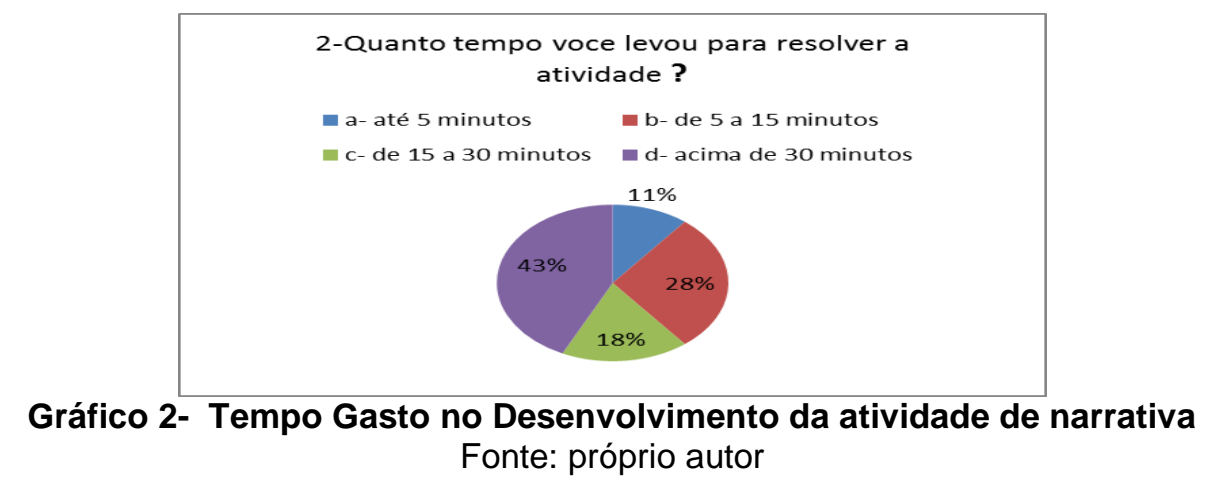

De acordo com o Gráfico 2, 43\% dos participantes levaram mais de 30 minutos para resolver a atividade proposta, destaca-se que o tempo gasto acima de 30 minutos se interliga a opinião dos alunos na pergunta anterior, onde a grande maioria considerou a atividade difícil. Ainda sobre a pesquisa, $28 \%$ dos alunos levaram entre 5 a 15 minutos para resolver a atividade, $11 \%$ resolveu a atividade em até 5 minutos, enquanto que $18 \%$ levaram de 15 a 30 minutos para a resolução do problema.

Como no estudo de caso anterior, foi feita uma pergunta aos estudantes sobre o objetivo da aula, se os mesmos compreenderam a atividade proposta.

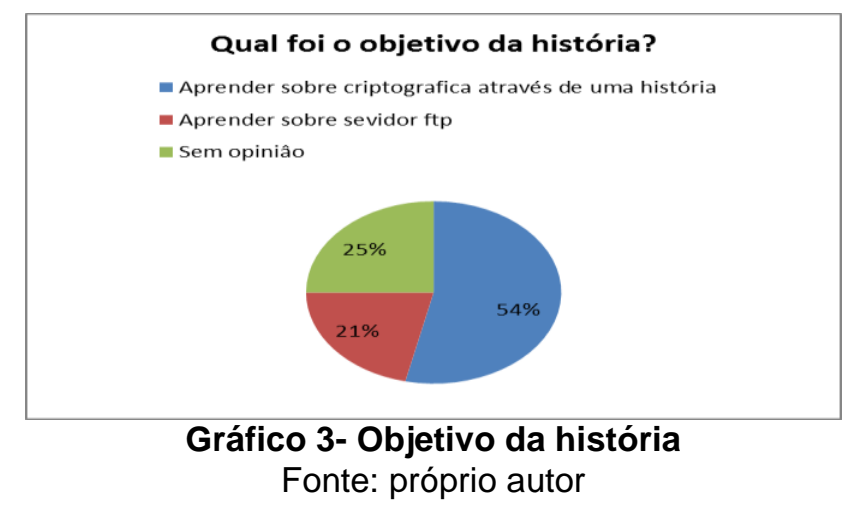

Observou-se no Gráfico 3 que 54\% dos participantes compreenderam o objetivo da atividade, que era contar uma história por meio de uma fábula, $21 \%$ entenderam que o objetivo da aula era a criação de um servidor de FTP e $25 \%$ não opinaram a respeito. É importante destacar que a atividade foi realizada por todos os alunos e mesmo a grande maioria achando a atividade muito difícil e demorada para ser finalizada, todos compreenderam e aprenderam sobre a proposta da aula. Notou-se uma motivação da turma em tentar solucionar as atividades propostas.

O estudo de caso trazia uma narrativa com características de OCC-RDD e transferia ao mestre, condutor do processo, o momento da aplicação de cenas "Objetivo", "Contratempo" e "Catástrofe", relembrando fatos, retornando quando necessária a algum momento da narrativa. 
Para a aplicação da técnica OCC-RDD é fundamental criar histórias, com cenas de "Contratempos" e "Catástrofes", que se conectem, ou seja, um determinado assunto precisa estar interligado a outro, essas "tramas" são essências para este modelo de aula, considerando também o tempo de execução para cada atividade e finalizar os exercícios com a devolutiva para os alunos.

As atividades apresentadas demonstraram-se eficiente sobre o ponto de vista do ensinoaprendizagem, pois, forneceram aos participantes do processo um conjunto de entendimento teóricos e práticos, que se fomentou com o desenvolvimento de suas habilidades.

\section{Conclusão}

Comprovou-se por meio do estudo de caso apresentado neste artigo, uma maior motivação dos alunos em tentar solucionar os problemas propostos e observou-se um equilíbrio cognitivo da sala, diante de cada abordagem definida por grau de aprendizagem. Observou-se que as cenas "Objetivo" não despertavam tanto o interesse dos estudantes, porém, ao serem desafiados principalmente nas cenas de "Catástrofe", os estudantes se sentiram parte do contexto da narração e motivados a chegar à resolução do problema.

Essa etapa do projeto demonstrou-se eficiente no ponto de vista da busca das melhores práticas pedagógicas, despertando a vontade de realizar raciocínios lógicos, trabalho em equipe e tomadas de decisões. Mas a pesquisa final, não se esgota apenas em fundamentar à técnica OCC-RDD e demonstrar estudos de caso, como também em criar um roteiro de aula semiautomático, com a ajuda de uma ferramenta desenvolvida a partir de uma máquina de estados que possa orientar o professor em suas aulas. Como resultado espera-se desenvolver tal ferramenta com base na técnica OCC-RDD que suporte a autoria de fábulas para um ambiente de aprendizagem interativo e presencial.

\section{Referências}

BATES, J. ; MUNDAY, S. Trabalhando com Alunos Superdotados, Talentosos e com Altas Habilidades. São Paulo, SP: $1^{\text {a }}$ ed. Galpão, 2007.

BLOOM, B. S. Taxonomy of Educational Objectives. Book 1: Cognitive Domain. New York, NY: $2^{\text {a }}$ ed. Longman, 1984.

MCCRAE. R., JOHN P. O. An Introduction to the Five-Factor Model and Its Applications. National Institute on Aging. WBI - the Workplace Bullying Institute, 1991. $<\mathrm{http}$ ://www.workplacebullying.org/multi/pdf/5factor-theory.pdf $>$ Maio 2014.

PAZETO, T. A.. PRIETCH, S. S. Estudo sobre a Evasão em um Curso de Licenciatura em Informática e Considerações para Melhorias. Anais do VIII Workshop de Educação e Informática Bahia- Alagoas, Sergipe- WEIBASE 2010, Maceió/AL. $<$ http://www.fejal.br/erbase2010/papers/weibase/65258.pdf $>$ Ago de 2014.

PERRAUDEAU, M. Estratégias de aprendizagem: Como acompanhar os alunos na aquisição dos saberes. Artmed Editora, 2006. Porto Alegre, RS.

VEGA, I. S. - Anotações feitas, fundamentadas pelas discussões do Prof, Dr. Ítalo S. Vega na orientação deste trabalho de mestrado. PUC-TIDD. SP. Realizado dia 14 de agosto de 2014, 15:30hs. 